\title{
Actualizaciones sobre "Irisina": la nueva mioquina
}

\author{
Irisin updates: \\ the new myokine
}

\begin{abstract}
In the continuous search of researchers to find an effective method to control obesity, a myokine called Irisin was found. Irisin is secreted mainly by skeletal muscle in response to exercise, either resistance, strength or high intensity, where High Intensity Interval Training (HIIT) is included. This polypeptide hormone acts mainly on subcutaneous adipose cells, turning white fat into brown fat. Brown fat is highly thermogenic, which enhance raising of total energy expenditure and helps maintaining or loosing corporal weight. Plasmatic Irisin levels are related positively with insulin sensitivity and weight loss. It has been also discovered that a greater plasmatic level of Irisin is related to the lengthening of telomeres, to a greater concentration of free tyrosine (T4) and it has been recently found that is related to an antitumoral effect on some types of cancer. All of the functions mediated by Irisin, have given it a protective action against different diseases, especially metabolic ones. The aim of this review was to update the knowledge about Irisin, and to show the effects that exercise has on its plasmatic levels, as well as comprehend how does the release of irisin influences different body systems. Counting with more information will allow the arising of new lines of investigation that will bring up non pharmacological therapeutic strategies for the treatment of non-communicable diseases.

Key words: Irisin, overweight, exercise, FNDC5, myokine.
\end{abstract}

\section{INTRODUCCIÓN}

De acuerdo a las estadísticas sanitarias mundiales publicadas por la Organización Mundial de la Salud (OMS) el año 2014, la obesidad es considerada como un factor de riesgo asociado al aumento de la morbilidad y mortalidad mundial (1). Las cifras de sobrepeso y obesidad a nivel mundial son preocupantes, alcanzando el sobrepeso u obesidad en niños menores de 5 años valores de 6,7\% (44 millones) mientras que la obesidad en adultos mayores de 20 años valores de $10 \%$ en hombres y el $14 \%$ en mujeres (2). En el caso de Chile las cifras son aún más alarmantes presentándose en niños menores de 5 años valores de 9,5\% de sobrepeso u obesidad, y en adultos mayores de 20 años 24,5\% de obesidad en hombres y 33,6\% en mujeres, lo que sitúa a nuestro país sobre los promedios mundiales (2).De acuerdo a los datos del Ministerio de Salud (MINSAL) existen 8,9 millones de chilenos con sobrepeso de los cuales 300 mil tienen obesidad y 1.200 .000
Luz María Trujillo G. $(1,2,4)$

Daniela García L. $(3,4)$

Astrid von Oetinger G. (3)

(1) Departamento de Morfofunción, Facultad de Medicina, Universidad Diego Portales, Santiago, Chile.

2) Escuela de Kinesiología, Facultad de Ciencias de la Salud, Universidad de Las Américas, Santiago, Chile. (3) Escuela de Kinesiología, Facultad de Ciencias de la Rehabilitación, Universidad Andrés Bello, Santiago, Chile. (4) Facultad de Medicina, Magister de Fisiología Clínica del Ejercicio, Universidad Mayor, Santiago, Chile

Dirigir la Correspondencia a: Klga.

Luz María Trujillo Gittermann, Departamento de Morfofunción, Facultad de Salud y Odontología,

Universidad Diego Portales,

Ejército 219, Santiago.

Teléfono: 56-226762256

Correo Electrónico: luzmariatrujillo.aga@gmail.com

Este trabajo fue recibido el 25 de Enero de 2016 y aceptado para ser publicado el 10 de Junio de 2016.

son diabéticos (3).

El aumento de la obesidad, asociado a estilos de vida no saludables, se ha convertido en un reconocido factor de riesgo de padecer diabetes tipo 2 (DM 2), hipertensión arterial (HTA), enfermedades cardiovasculares, y algunos tipos de cáncer (4).

Entidades y estudios científicos, han mostrado que el ejercicio es un estímulo potente para mejorar y prevenir la obesidad así como también las comorbilidades asociadas a ella $(5,6)$. Por esta razón, es que hace algunos años los investigadores se han focalizado en encontrar maneras de hacer que las actividades deportivas sean más eficientes en el control de peso y de las comorbilidades asociadas a la obesidad y sedentarismo (6).

Es en esta búsqueda continua por combatir de manera más efectiva la obesidad que Boström y cols, 2012, descubre una mioquina Ilamada Irisina, la que se expresa en el tejido 
músculo esquelético (7).

Existe evidencia científica de que la Irisina se relaciona positivamente con la sensibilidad a la insulina y la pérdida de peso (7). Además está involucrada en el proceso de conversión de tejido adiposo blanco a tejido adiposo pardo $(7,8)$, el que es altamente termogénico y se ha propuesto como como un potencial tratamiento contra la obesidad $(9,10)$.

Se ha demostrado que la Irisina aumenta sus niveles plasmáticos con el entrenamiento y estos resultados proveen la evidencia suficiente para decir que la Irisina podría mediar alguno de los posibles efectos benéficos del ejercicio (7).

Esta revisión tuvo por objetivo actualizar el conocimiento referente a la Irisina, evidenciando los efectos que la realización de ejercicio tiene sobre los niveles plasmáticos de ésta, así como también comprender como su liberación influye en distintos sistemas corporales. El contar con mayor información dará paso a nuevas líneas de investigación y permitirá contar con estrategias terapéuticas no farmacológicas que contribuyan en el tratamiento de enfermedades crónicas no transmisibles.

\section{MATERIAL Y METODO}

El presente estudio es una revisión de tipo bibliográfica narrativa donde se incluyeron 61 artículos científicos, de los cuales, 56 son artículos originales, 2 revisiones bibliográficas y 3 metaanálisis. Los artículos incluidos fueron obtenidos de la base de dato Pubmed, escritos en idioma inglés, realizados en humanos (58 artículos) y animales (3 artículos), y publicados entre los años 1997 y 2015.

\section{IRISINA: LA NUEVA MIOQUINA}

El año 2012 Boström y cols. (7), descubren una mioquina noble a la que llamó Irisina, por la diosa griega de la mensajería "IRIS". La Irisina es una hormona polipeptídica pequeña de 112 aminoácidos, secretada como producto de la fibronectina tipo III teniendo en su dominio la proteína 5 (FNDC5) y es inducida por el receptor activado por proliferador de peroxisomas $y$ (PPARY) y el coactivador transcripcional $1 \alpha(P G C-1 \alpha)$ en el músculo esquelético (11).

Se le atribuye a la Irisina el rol de mediar alguna de las ventajas que se conocen del ejercicio físico, encontrándose como principal función la de actuar sobre células adiposas subcutáneas, transformando "grasa blanca en grasa parda", la que es altamente termogénica, por medio del aumento de la expresión de la proteína de desacoplamiento mitocondrial 1 (UCP1) (12-14). A su vez, tiene el rol de ser mediador de otros de los efectos benéficos del ejercicio como son el aumento de la biogénesis mitocondrial y del metabolismo oxidativo (15).

\section{SECRECIÓN DE IRISINA}

El músculo esquelético es un órgano endocrino que juega un rol importante en la homeostasis metabólica gracias a la producción de proteínas denominadas mioquinas, que actúan como hormonas y cuya liberación está relacionada con la contracción de la fibra muscular $(16,17)$.

Boström y cols. 2012, determinaron que la secreción de Irisina es ejercicio dependiente y por lo mismo le otorgan a esta proteína la denominación de mioquina (7).

Estudios más recientes han reconocido otros sitios de secreción de la Irisina como son: el tejido adiposo subcutáneo y visceral $(18,19)$, las glándulas salivares (20), piel, hígado, riñón y músculo cardíaco (21).

El año 2015, Aydin y cols. obtienen evidencia sugerente de que la principal fuente de Irisina no sería el sarco- plasma del músculo esquelético sino que en las capas de los nervios que se extienden en el músculo esquelético. Además, encuentran que habrían otros potenciales sitios de secreción diferentes de los ya mencionados como son testículos, páncreas, bazo, cerebro y estómago (13).

\section{IRISINA Y TRASTORNOS METABÓLICOS}

La obesidad se asocia con un mayor riesgo de adquirir distintas patologías, y aumentando la morbilidad y mortalidad (22). Se ha determinado que por las características en la distribución de grasa corporal, es la obesidad de tipo genoide, también llamada obesidad central, la que estaría más relacionada con el riesgo cardiovascular y de sufrir otras patologías (23-25).

Por ésta razón los autores se han centrado en estudiar las características de los adipocitos, determinando que el tejido adiposo blanco almacena energía (triglicéridos) mientras que los adipocitos pardos consumen energía $(26,27)$.

La grasa parda juega un papel fundamental en la termogénesis y el gasto energético en los lactantes, y si bien hace algunos años se pensaba que en los adultos ésta no estaba presente, actualmente y gracias a las nuevas tecnologías se ha podido evidenciar lo contrario, y se ha podido determinar que su concentración está asociada a la cantidad de masa magra ya que su liberación es mediada por la contracción muscular (28).

Distintos estudios han mostrado que los cambios en la actividad del tejido adiposo pardo pueden afectar la termogénesis y la homeostasis de la glucosa. La capacidad termogénica de la grasa parda está mediada por la presencia de la UPC 1 cuya expresión está regulada por varios factores transcripcionales incluyendo el PPARy y PGC1 $\alpha$, los que pueden ser inducidos por exposición al frío y al ejercicio a través de la función de la Irisina (figura 1) $(28,29)$. El aumento en la expresión del UPC 1 genera, como uno de sus efectos, el pardeamiento del tejido adiposo blanco transformándolo en tejido adiposo pardo termogénicamente más activo, lo que aumenta el gasto energético total $(30,31)$. El tejido adiposo pardo contiene un alto número de partículas lipídicas y un alto número de mitocondrias $(32,33)$. En el interior de la membrana mitocondrial se encuentra la UPC 1, una proteína desacoplante, que al activarse no genera síntesis de ATP sino que produce un aumento en la liberación de temperatura $(34,35)$. Por estas razones la Irisina es considerada una proteína termogénica que aumenta el gasto energético al ser una hormona que media la transformación del tejido adiposo blanco a pardo (7).

Estudios realizados en sujetos con obesidad mórbida han demostrado una asociación inversa entre el IMC y las concentraciones plasmáticas de Irisina $(36,37)$. A su vez, en los pacientes que han perdido peso por cirugía bariátrica, se ha encontrado una disminución en los niveles de Irisina plasmática (36). Esto se debe a que la cantidad de masa magra tiene directa relación con la cantidad de Irisina liberada, y por eso, aquellos sujetos que bajan de peso disminuyendo su porcentaje de masa muscular presentan disminución en las concentraciones de Irisina $(36,37)$.

Gutiérrez y cols. el año 2014 (38), realizaron un estudio comparando sujetos obesos mórbidos con sujetos normopeso, determinando que los sujetos normopeso presentan concentraciones más altas de Irisina. Además, descubren que la concentración de Irisina depende del tipo de obesidad, siendo los sujetos con obesidad de tipo androide los que tienen concentraciones más bajas de Irisina comparados con los de obesidad genoide. Estas diferencias se mantuvieron incluso después que los sujetos con obesidad mórbida fueron some- 
tidos a cirugía bariátrica (38). En este mismo estudio luego de 24 horas de incubación in vitro con leptina se observó una disminución significativa de la expresión del FNDC5 mRNA, precursor de Irisina, determinándose que la leptina podría estar involucrada en la regulación de ésta (38).

Como mencionamos anteriormente la obesidad se relaciona con el aumento de posibilidades de sufrir otra patologías, siendo la diabetes tipo 2 una de ellas $(4,22)$. Por lo mismo decidimos ver si existía literatura que relacionara la DM 2 con la Irisina, encontrando el estudio de Liu y cols, quienes el año 2013, reportaron que las concentraciones plasmáticas de Irisina son significativamente menores en los diabéticos tipo 2, y una relación positiva entre las concentraciones de Irisina y el IMC, la pérdida de peso y la sensibilidad a la insulina, sugiriendo que la Irisina puede jugar un rol crucial en la intolerancia a la glucosa y en la diabetes tipo 2 (39). Lo encontrado en este artículo concuerda con el estudio presentado por Choi y cols (40), sin embargo, se contrapone con lo reportado en otros artículos donde se encontró que la Irisina circulante se asocia positivamente con la resistencia a la insulina y a los niveles de glucosa sanguínea post prandial en sujetos no diabéticos (41-44). Esto indicaría que la Irisina está involucrada en la homeostasis de la glucosa (44), y explicaría que el aumento en la liberación de Irisina ocurre como mecanismo compensatorio tanto por el tejido adiposo como muscular con el fin de regular la deteriorada función de la insulina (41).

\section{IRISINA Y ENVEJECIMIENTO}

Se ha encontrado una correlación negativa entre las concentraciones de Irisina y la edad $(p=0,001)$, a medida que pasan los años es menor la liberación de Irisina, lo que se atribuye a la pérdida fisiológica de la masa muscular propia del envejecimiento (36).

El año 2012, Osthus y cols, descubrieron que la Irisina liberada por el músculo al realizar ejercicio de resistencia favorece la neurogenesis, determinante del crecimiento de nuevas células nerviosas (45). A lo largo de los años se le han seguido atribuyendo beneficios a la realización de ejercicio regular como es la mejora de la función cognitiva, y se cree que la Irisina sería la responsable de mediar este proceso, protegiendo al cerebro contra la degeneración (46). También se ha determinado que la Irisina aumenta la expresión del factor neurotrófico derivado del cerebro (BDNF), asociado a las capacidades de aprendizaje, memoria y el proceso de envejecimiento (30).

Rana y cols, el año 2014, demostraron que la Irisina desacelera el proceso de envejecimiento mediante alargamiento de los telómeros. Demostraron que existe una relación significativa entre los niveles de Irisina en sangre y un marcador biológico de envejecimiento relacionado con la longitud de los telómeros, llegando a la conclusión de que las personas que tienen niveles más altos Irisina resultaron ser "biológicamente más joven" que aquellos con niveles más bajos de la hormona (47).

\section{FIGURA 1}

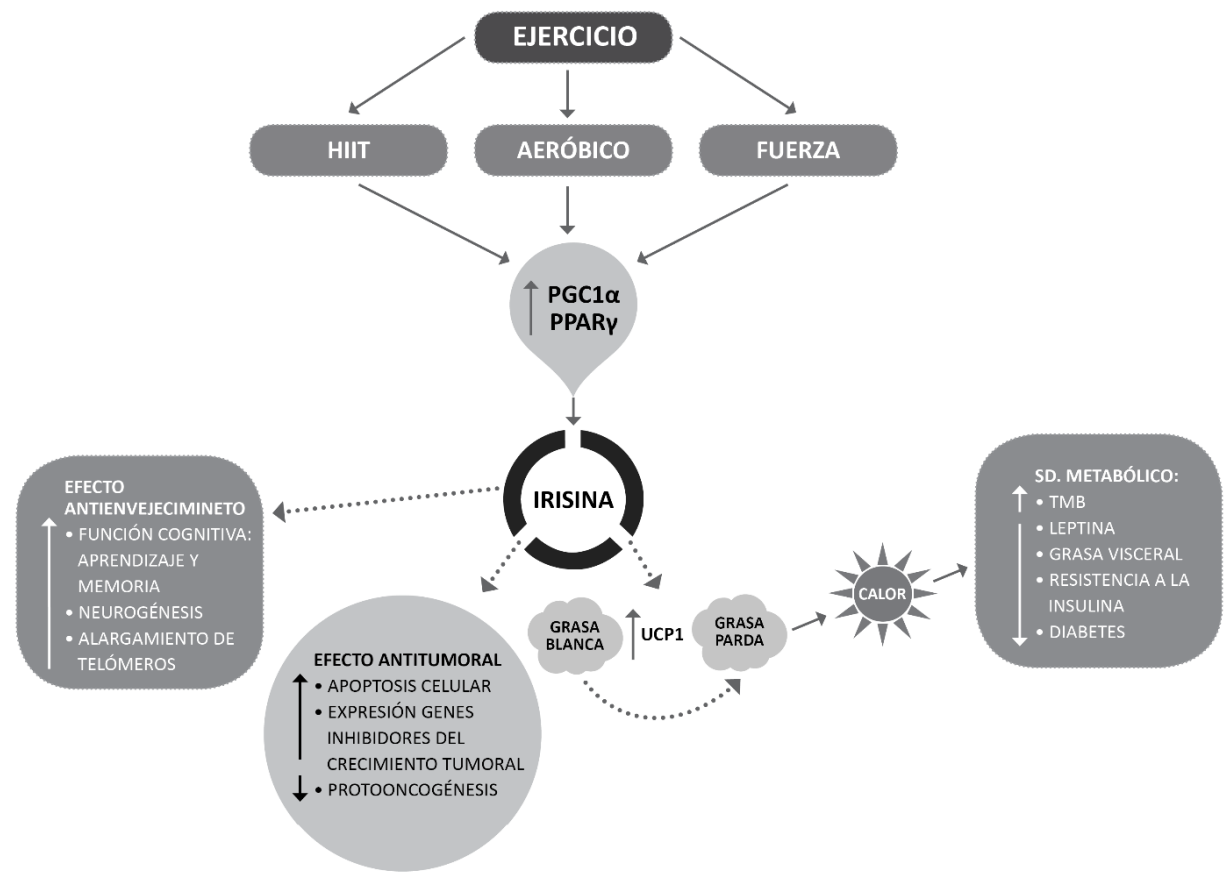

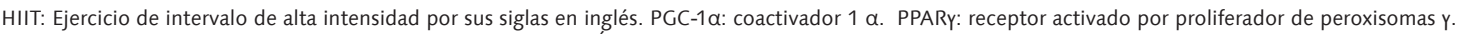

UCP1: Proteína de desacoplamiento mitocondrial 1. SD.METABÓLICO: Síndrome metabólico. TMB: Tasa Metabólica Basal. 


\section{IRISINA Y FUNCIÓN TIROIDEA}

La Irisina secretada por los miocitos cumple la función de transmitir señales desde el músculo esquelético a otros tejidos. Investigaciones han reportado la influencia significativa que tiene sobre el metabolismo y la termogénesis $(30,48,49)$. Sin embargo, hay otros factores que influyen en el estado metabólico como lo son las hormonas tiroideas, aumentando la termogénesis, el balance energético y también actuando sobre el tejido adiposo pardo $(50,51)$.

Ruchala y cols, en el año 2014, relacionaron las concentraciones de Irisina plasmáticas con la función y producción de hormonas tiroideas, demostrando que los niveles de Irisina son menores en pacientes con hipotiroidismo. Determinaron que existe una correlación positiva entre T4 libre y la concentración de Irisina plasmática, así también, que los pacientes que presentaban marcadores de daño muscular más elevados, estudiados mediante los niveles de creatina kinasa (CK), tenían menores concentraciones de Irisina plasmática (52).

\section{IRISINA Y CÁNCER}

Los estudios en relación a los efectos de la Irisina y el cáncer son incipientes y limitados. El año 2014, Moon y cols, realizaron un estudio sin lograr demostrar efectos de la Irisina sobre la proliferación celular y potencial malignidad de células cancerígenas relacionadas con la obesidad (53).

El año 2014, el estudio de Gannon y cols, los hallazgos sugieren que la Irisina tiene un efecto supresor en el número de células y características migratorias de las células malignas de cáncer de mama; así como también demostraron que es capaz de inducir apoptosis en dichas células (54).

El año 2015 a partir del estudio de Agniezka y cols, nuevamente demuestran efectos benéficos de la Irisina como potencial agente anti tumoral. En los potenciales efectos destacan el aumento en apoptosis celular, detención de la migración celular e invasión a través de la matriz extracelular, aumento de la expresión de genes asociados con la inhibición del crecimiento tumoral, así como también la disminución en la expresión de algunos proto oncogenes (genes normales que se transforman en cancerígenos debido a mutaciones o aumentos anormales de su expresión) (55).

\section{IRISINA Y EJERCICIO}

Se ha relacionado a la Irisina como el potencial mediador de algunos de los beneficios metabólicos que genera el ejercicio físico, ya que es el ejercicio quién induce la expresión de PGC -1 $\alpha$, la que induce liberación de Irisina al torrente sanguíneo (figura 1) (56-58).

En estudios que evidencian estos beneficios metabólicos se muestra el de Lecker y cols, 2012, quién determinaron que los pacientes con insuficiencia cardíaca que poseen una mejor capacidad aeróbica tras ser evaluados mediante consumo máximo de oxígeno (VO2 máx), presentan niveles de Irisina más elevados que aquellos con menor VO2 máx (48). Ese mismo año, Huh y cols, revelaron que los niveles circulantes de Irisina en sujetos obesos aumentan después de 30 minutos de ejercicio agudo y se correlacionan positivamente con nivel de ATP, metabolitos relacionados a lipolisis y glucólisis en el músculo, así como también, con la circunferencia de bíceps, siendo, el predictor más fuerte de los niveles circulantes de Irisina (36).

Es relevante entender cómo los distintos tipos de ejercicio influyen en la liberación de Irisina. Bostrom y cols, el año 2012, demostraron que los niveles de Irisina aumentan como resultado de la realización de ejercicio aeróbico regular, así también, de diferentes protocolos de ejercicios de alta intensidad (7).

Tsuchiya y cols, el año 2014, compararon un protocolo de alta intensidad al $80 \%$ del VO2 máx, versus un protocolo de baja intensidad al 40\% del VO2 máx, obteniendo como resultado que la respuesta de liberación de Irisina depende de la intensidad del ejercicio, ya que el grupo de ejercicio de alta intensidad, quienes mostraron niveles de lactato más altos, presentaron concentraciones plasmáticas de Irisina más altas que sus nivele de pre ejercicio, y en relación al grupo de ejercicio de baja intensidad (31).

Tsuchiya y cols, en Mayo 2015 decidieron comparar la respuesta de la Irisina frente al ejercicio de resistencia, resistido y a un protocolo mixto. Los resultados arrojaron que en el protocolo de ejercicio de fuerza la respuesta de la Irisina fue significativamente mayor $(\mathrm{P}<0,05)$ comparado con los otros dos protocolos (59).

Nygaard y cols. 2015, demostraron que una sola sesión de ejercicio podría aumentar las concentraciones de Irisina circulante, y por lo mismo evaluar la respuesta aguda de un ejercicio de resistencia de tipo interválico de alta intensidad y de un ejercicio de fuerza intenso. Lo que se determinó fue que en ambos protocolos de ejercicio la concentración de Irisina en sangre aumentó (60). Estos hallazgos confirman lo planteado por otros autores que postulan que la Irisina es una mioquina inducida por el ejercicio y que su aumento es transitorio $(61,62)$.

Qiu y colaboradores, en su metaanálisis (63), analizaron el efecto del entrenamiento crónico en los niveles de Irisina circulante en población adulta, el estudio incluyó 8 artículos, de los cuales dos, de tipo randomizados controlados $(63,64)$ obtuvieron resultados concluyentes; determinando que el entrenamiento crónico disminuye significativamente $(p<0,05)$ los niveles de Irisina circulantes, esto se explicaría por qué la Irisina no sólo es una mioquina sino que también una adipoquina, entonces al entrenar de manera crónica cambia la composición corporal incluido el porcentaje de grasa, haciendo posible que la Irisina disminuya $(18,19,66,67)$. Sin embargo, al analizar los datos aislados del grupo entrenado (antes y después) se encontró que los niveles circulantes de Irisina estaban significativamente aumentados después del entrenamiento crónico (65) hallazgos consistentes con lo inicialmente reportado por Bostrom y cols. (7)

\section{CONCLUSIONES}

La evidencia analizada respalda el hecho de que la liberación de Irisina es mediada por el entrenamiento, los estudios plantean que los distintos tipos de ejercicio favorecen su liberación al torrente sanguíneo, pero existe una mayor liberación cuando los ejercicios son de alta intensidad o de fuerza.

Si bien faltan estudios que puedan ofrecer claridad sobre qué protocolos de ejercicios son más eficientes para promover la liberación de Irisina, existe bastante evidencia analizada en esta revisión que respalda que esta mioquina es la encargada de mediar muchos de los beneficios atribuibles al ejercicio, teniendo incidencia en patologías como el cáncer y diabetes tipo 2, en el proceso de envejecimiento, y no sólo en la termogénesis como se creía en un principio (figura 1).

Por lo anteriormente mencionado, consideramos de gran utilidad contar con estudios realizados en poblaciones más numerosas y que utilicen distintos protocolos de ejercicios a fin de poder recoger información que permita generar estrategias más efectivas que potencien la liberación de ésta mioquina para así poder contar con nuevas herramientas terapéu- 
ticas no farmacológicas para el tratamiento de enfermedades crónicas no transmisibles.

\section{RESUMEN}

En la búsqueda continua de los investigadores por combatir de manera más efectiva la obesidad, se descubre una mioquina llamada Irisina. La Irisina es secretada principalmente por el músculo esquelético en respuesta al ejercicio, ya sea aeróbico, de fuerza o de alta intensidad, donde se incluyen, ejercicios de intervalo de alta intensidad (HIIT). Esta hormona polipeptídica actúa principalmente sobre células adiposas subcutáneas, transformando grasa blanca en grasa parda. La grasa parda es altamente termogénica, lo que favorece el aumento del gasto energético total y ayuda a mantener o incluso a perder peso corporal. La concentración de Irisina plasmática se relaciona positivamente con la sensibilidad a la insulina y la pérdida de peso. Además, se ha descubierto que una mayor concentración de Irisina plasmática se relaciona con el alargamiento de los telómeros, y también, con una mayor concentración de T4 libre y con un recién descubierto efecto antitumoral en algunos tipos de cáncer. Todas las funciones mediadas por la Irisina, le atribuyen una acción protectora contra distintas enfermedades, especialmente metabólicas. El objetivo de esta revisión fue actualizar el conocimiento sobre la Irisina, evidenciando los efectos que tiene la realización de ejercicio sobre los niveles plasmáticos de ésta, así como también comprender como su liberación influye en distintos sistemas corporales. El contar con mayor información dará paso a nuevas líneas de investigación y permitirá contar con estrategias terapéuticas no farmacológicas que contribuyan en el tratamiento de enfermedades crónicas no transmisibles.

Palabras clave: Irisina, obesidad, ejercicio, FNDC5, mioquinas.

\section{BIBLIOGRAFÍA}

1. WHO. World Health Statistics 2014. Available at: http://apps.who.int/iris/bitstream/10665/131953/ 1/9789240692695_spa.pdf?ua=1 (page 116-117, Retrieved Nov. 18, 2015).

2. WHO. World Health Statistics 2014. Available at: http://apps.who.int/iris/bitstream/10665/131953/ 1/9789240692695_spa.pdf?ua=1 (page 118-126, Retrieved Nov. 18, 2015).

3. MINSAL. Ministry of Health. Chile's goverment. National Health Survey 2009-2010 ENS Chile. Chile 2011. Available at:http://web.minsal.cl/portal/url/item/bcb03d7bc28b64dfe040010165012d23.pdf (Retrieved Nov. 18, 2015).

4. WHO. World Health Organization. The World Health Report 2022. Reducing Risks, Promoting Healthy Life. WHO 2012. Disponible en: http://www. who.int/whr/2002/en/ whr02_en.pdf (Consultado el 16 de Agosto de 2014).

5. American College of Sports Medicine. Exercise prescription. In: ACSM's Guidelines for Exercise Testing and Prescription (7th Ed.), Edited by Whaley MH. Baltimore, MD: Lippincott Williams \& Wilking, 2006.

6. Haskell WL, Lee IM, Pate RR, Powell KE, Blair SN, Franklin BA, Macera CA, Heath GW, Thompson PD, Bauman A. Physical Activity and Public Health: Updated Recommendation for Adults From the American College of Sports Medicine and the American Heart Association. Circulation. 2007;116:1081-93.

7. Boström $P, W u$ J, Jedrychowski MP, Korde A, Ye L, Lo JC, Rasbach KA, Boström EA, Choi JH, Long JZ, Kajimura S,
Zingaretti MC, Vind BF, Tu H, Cinti S, Hojlund $K$, Gygi SP, Spiegelman BM.A PGC1- $\alpha$-dependent myokine that drives brown-fat-like development of white fat and thermogenesis. Nature 2012; 481: 463-8.

8. Fisher FM, Kleiner S, Douris N, Fox EC, Mepani RJ, Verdequer F, Wu J, Kharitonenkov A, Flier JS, Maratos-Flier E, Spiegelman BM. FGF21 regulates PGC-1a and browning of white adipose tissues in adaptive thermogenesis. Genes Dev 2012; 26: 271-81.

9. Wu J, Boström P, Sparks LM, Ye L, Choi JH, et al. Beige adipocytes are a distinct type of thermogenic fat cell in mouse and human. Cell 2012; 150: 366-76.

10. Saito M, Okamatsu-Ogura Y, Matsushita M, Watanabe $K$, Yoneshiro T, et al. Brown Fat in Humans: Turning up the Heat on Obesity. Diabetes 2009; 58: 1482-4.

11. Kelly DP. Irisin, Light my fire. Science 2012;336:42-3.

12. Shan T, Liang $X, B I P$, Kuang $S$. Myostatin Knockout drives browning of white adipose tissue through activating the AMPK-PGC1alpha-Fndc5 pathway in muscle. FASEB J 2013;27:1981-9.

13. Aydin S, Kuloglu T, Aydin A, Kalayci M, Yilmaz M, Cakmak T, Albayrak S, Gungor S, Colakoglu N, Ozercan I. A comprehensive immunohistochemical examination of the distribution of the fat-burning protein irisin in biological tissues. Peptides 2014;61:130-6.

14. Aydin S.Three new players in energy regulation: Preptin, adropin and irisin. Peptides 2014; 56: 94-110.

15. Handschin C, Spiegelman BM.The role of exercise and PGC1a in inflammation and chronic disease. Nature 2008; 454: 463-9.

16. Pratesi A, Tarantini F, Di Bari M. Skeletal Muscle: an endocrine organ. Clin Cases Muner Bone Metab. 2013; 10:11-4.

17. Brandt C, Pedersen BK. The role of exercise-induced myokines in muscle homeostasis and the defense against chronic disease. J Biomed. Biotechnol. V 2010; Article ID 520:258, 6 pages.

18. Roca-Rivada A, Castelao C, Senin LL, Landrove MO, Baltar $J$, et al. FNDC5/Irisin Is Not Only a Myokine but Also an Adipokine. PLoS ONE 2013; 8(4): e60563.

19. Moreno-Navarrete JM, Ortega F, Serrano M, Guerra $E_{\text {, }}$ Pardo G, Tinahones F, Ricart W, Fernandez-Real JM. Irisin is expressed and produced by human muscle and adipose tissue in association with obesity and insulin resistance, "J Clin Endocrinol Metab. 2013; 98(4):E769 -E78

20. Handschin C, Spiegelman BM. The role of exercise and PGC1a in inflammation and chronic disease. Nature 2008; 454:463-9.

21. Aydin S, Kuloglu T, Aydin S, Eren MN, Celik A, Yilmaz M, et al. Cardiac, skeletalmuscle and serum irisin responses to with or without water exercise in Young and old male rats: cardiac muscle produces more irisin than skeletal muscle. Peptides 2013;52C:68-73.

22. World Health Organization. Waist Circumference and Waist-hip Ratio. Report of a WHO Expert Consultation. Generve: WHO; 2011.

23. Yusuf S, Hawken S, Ounpuu S, Bautista L, Franzosi MG, Commerford $P$, Lang CC, Rumboldt Z, Onen CL, Lisheng L, Tanomsup S, Wangai P Jr, Razak F, Sharma AM, Anand SS. Obesity and the risk of myocardial infarction in 27,000 participants from 52 countries: a case-control study. Lancet 2005;366:1640-9.

24. Czernichow S, Kengne AP, Stamatakis E, Hamer M, Batty $G D$. Body mass index, waist circumference and waist-hip ratio: which is the better discriminator of cardiovascular 
disease mortality risk?: evidence from an individualparticipant meta-analysis of 82864 participants from nine cohort studies. Obes Rev. 2011;12:680-7.

25. Sanchis-Gomar F, Pérez-Quilis C. Irisinemia: a novel concept to coin in clinical medicine? Ann Nutr Metab. 2013;63:60-1.

26. Cannon B, Nedergaard J. Brown adipose tissue: function and physiological significance. Physiol Rev. 2004;84: 277-359.

27. Cinti S. Adipocyte differentiation and transdifferentiation: plasticity of the adipose organ. J Endocrinol Invest. 2002; 25:823-35.

28. Nedergaard J, Bengtsson T, Cannon B. Unexpected evidence for active brown adipose tissue in adult humans. Am J Physiol Endocrinol Metab. 2007;293:444 - 52.

29. Barbera MJ, Schluter A, Pedraza N, Iglesias $R$, Villarroya $F$, Giralt M. Peroxisome proliferator-activated receptor alpha activates transcription of the brown fat uncoupling protein-1 gene. A link between regulation of the thermogenic and lipid oxidation pathways in the brown fat cell. J Biol Chem. 2001;276:1486-93.

30. Zhang $Y$, Li R, Meng Y, Li S, Donelan W, Zhao Y, Qi L, Zhang $M$, Wang $X$, Cui T, Yang $L, i$ Tang $D$. Irisin Stimulates Browning of White Adipocytes Through Mitogen-Activated Protein Kinase p38 MAP Kinase and ERK MAP Kinase Signaling.Diabetes 2014;63:514-25.

31. Tsuchiya $Y$, Ando D, Goto K, Kiuchi M, Yamakita M, Koyama K. High-Intensity Exercise causes Greater Irisin Response Compared With Low-Intensity Exercise under Similar Energy Consumption. Tohoku J Exp Med. 2014; 233:135 - 40.

32. Enerbäck S. Brown adipose tissue in humans. IntJ Obes (Lond) 2010;34(Suppl.1):S43-6.

33. Lidell ME, Enerbäck S. Brown adipose tissue - a new role in humans. Nat Rev Endocrinol. 2010;6:319-25.

34. Aydin S. Presence of adropin, nesfatin-1, apelin-12, ghrelins and salusins peptides in the milk, cheese whey and plasma of dairy cows. Peptides 2013;43:83-7.

35. Jastroch M, Divakaruni AS, Mookerjee S, Treberg JR, Brand MD. Mitochondrial proton and electron leaks. Essays Biochem. 2010;47:53-67.

36. Huh JY, Panagiotou G, Mougios V, Brinkoetter M, Vamvini MT, Schneider BE, Mantzoros CS. FNDC5 and irisin in humans: I. Predictors of circulating concentrations in serum and plasma and II. mRNA expression and circulating concentrations in response to weight loss and exercise. Metabolism 2012;61:1725-38.

37. Stengel A, Hofmann T, Goebel-Stengel M, Elbelt U, Kobelt $P$, Klapp BF. Circulating levels of irisin in patients with anorexia nervosa and different stages of obesity-correlation with body mass index. Peptides 2013;39:125-30.

38. Gutierrez C, Garcia S, Rodrigez F, Garcia E, Haro J, Garcia J, Valdes S, Gonzalo M, Soriguer F, Moreno F, Rodriguez A, Martinez A, Santoyo J, Perez V, Garcia E. FNDC5 could be regulated by leptin in adipose tissue. Eur J Clin Invest. 2014;44(10): 918-25.

39. Liu jj, Wong MD, Toy WC, Tan CS, Liu S, NG XW, Tavintharan S, Sum CF, Lim SC. Lower circulating irisin is associated with type 2 diabetes mellitus. J Diabetes Complications 2013;27:365-69.

40. Choi YK, Kim MK, Bae KH, Jeong JY, Lee WK, Kim JG, Lee $I K$, Park KG. Serum irisin levels in new onset type 2 diabetes. Diabetes Res Clin Pract. 2013; 100:96-101.

41. Park KH, Zaichenko L, Brinkoetter M, Thakkar B, Sahin-Efe
A, Joung KE, Tsoukas MA, Geladari EV, Huh JY, Dincer $F$, Davis CR, Crowell JA, Mantzoros CS. Circulating irisin in relation to insulin resistance and the metabolic syndrome. J Clin Endocrinol Metab. 2013;98(12):4899-907.

42. Sesti G, Andreozzi F, Fiorentino TV, Mannino GC, Sciacqua A, Marini MA, Perticone F. High circulating irisin levels are associated with insulin resistance and vascular atherosclerosis in a cohort of nondiabetic adult subjects. Acta Diabetol. 2014;51(5):705-13.

43. Garcés MF, Peralta JJ, Ruiz-Linares CE, Lozano AR, Poveda NE, Torres-Sierra AL, Eslava-Schmalbach JH, Alzate JP, Sánchez AY, Sanchez E, Angel-Müller E, Ruíz-Parra Al, Diéguez $C$, Nogueiras R, Caminos JE. Irisin levels during pregnancy and changes associated with the development of preeclampsia. J Clin Endocrinol Metab. 2014;99(6):2113-9.

44. Al-Daghri NM, Alkharfy KM, Rahman S, Amer OE, Vinodson B, Sabico S, Piya MK, Harte AL, McTernan PG, Alokail MS, Chrousos GP. Irisin as a predictor of glucose metabolism in children: sexually dimorphic effects. Eur J Clin Invest. 2014;44(2):119-24.

45. Osthus IB, Sgura A, Berardinelli F, Alsnes IV, Bronstad E, Rehn T, Stobakk PK, Hatle H, Wisloff U, Nauman J.Telomere length and long-term endurance exercise: does exercise training affect biological age? A pilot study. PLoS One 2012, 7:e52769.

46. Sharma N, Castorena CM, Cartee GD. Greater insulin sensitivity in calorie restricted rats occurs with unaltered circulating levels of several important myokines and cytokines. Nutr Metab (Lond) 2012; 9:90.

47. Rana KS, Arif M, Hill EJ, Alfred S, Nagel DA, Nevill A, Randeva HS, Bailey CJ, Bellary S, Brown JE. Plasma Irisin levels predict telomere length in healthy adults. Age 2014;36:995-1001.

48. Lecker SH, Zavin A, Cao P, Arena R, Allsup K, Daniels KM, Joseph J, Schulze $P C$, Forman DE. Expression of the irisin precursor FNDC5 in skeletal muscle correlates with aerobic exercise performance in patients with heart failure. Circ Heart Fail. 2012;5:812-8.

49. Raschke S, Eckel J. Adipo-myokines: two sides of the same coin-mediators of inflammation and mediators of exercise. Mediators Inflamm V 2013, Article ID 320724, 16 pages.

50. Fisher $J N$, Ball EG. Studies on the metabolism of adipose tissue. $X X$. The effect of thyroid status upon oxygen consumption and lipolysis. Biochemistry 1967;6:637-47.

51. Silva JE. Thyroid hormone control of thermogenesis and energy balance. Thyroid 1995;5:481-92.

52. Ruchala M, Zybek A, Szczepanek-Parulska E.Serum irisin levels and thyroid function-Newly discovered association. Peptides 2014; 60: 51-5.

53. Moon HS, Mantzoros CS, Regulation of cell proliferation and malignant potential by irisin in endometrial, colon, thyroid and esophageal cancer cell lines. Metabolism 2014; 63 (2): 188-93.

54. Gannon NP, Roger A. Vaughan RA, Garcia-Smith $R$, Marco Bisoffi M, Trujillo KA. Effects of the exercise-inducible myokine irisin on malignant and non-malignant breast epithelial cell behavior in vitro. Int J Cancer 2015; 136: E197- 202.

55. Mazur-Bialy Al, Oplawski M, Wypasek E, Zarawski M. Irisin - A newly discovered adipomiokine - Impairs growth and progression of breast cancer MDA-MB-231 cell line. Cytokine, 2015; 76:107.

56. Cunha A. basic research: Irisin Behind the benefits of ex- 
ercise. Nar Rev Endocrinol. 2012; 8: 195.

57. Pekkala S, Wiklund PK, Hulmi JJ, Ahtiainen JP, Horttanainen $M$, Pollanen $E$, et al. Are skeletal muscle FNDC5 gene expression and irisin release regulated by exercise and related to health? J Physiol. 2013;591:5393-400.

58. Timmons JA, Baar K, Davidsen PK, Atherton PJ. Is irisin a human exercise gene? Nature 2012;488. E9-10; discussion $E-1$.

59. Tsuchiyaa Y, Ando D, Takamatsuc K, Goto K. Resistance exercise induces a greater irisin response than endurance exercise. Metabolism 2015; 64: 1042-50.

60. Nygaard H, Slettaløkken G, Vegge $G$, Hollan I, Whist JE, Strand T, Rønnestad BR, Ellefsen S. Irisin in Blood Increases Transiently after Single Sessions of Intense Endurance Exercise and Heavy Strength Training. PLOS ONE 2015; 10(3): e0121367.

61. Huh JY, Mougios V, Kabasakalis A, Fatouros I, Siopi A, Douroudos II, Filippaios A, Panagiotou G, Park KH, Mantzoros CS. Exercise-induced irisin secretion is independent of age or fitness level and increased irisin may directly modulate muscle metabolism through AMPK activation. J Clin Endocrinol Metab. 2014:jc20141437.

62. Kraemer RR, Shockett P, Webb ND, Shah U, Castracane VD. A transient elevated irisin blood concentration in response to prolonged, moderate aerobic exercise in young men and women. Hormone and metabolic research $=$ Hormon- und Stoff wechselforschung $=$ Hormones et metabolisme. 2014; 46 (2):150-4.

63. Qiu S, Cai X, Sun Z, Schumann U, steinacker JM. Chronic exercise training and circulating irisin in adults: a Meta - Analysis. Sport Med 2015; DOI 10.1007/s40279-0140293-4.

64. Hecksteden A, Wegmann M, Steffen A, Kraushaar J, Arne Morsch A, Ruppenthal S, Kaestner L, Meyer T.Irisin and exercise training in humans: results from a randomized controlled training trial. BMC Med. 2013;11:235.

65. Scharhag-Rosenberger F, Meyer T, Wegmann M, Ruppenthal S, Kaestner L, Morsch A, Hecksteden A. Irisin does not mediate resistance training-induced alterations in resting metabolic rate. Med Sci Sports Exerc. 2014;46(9):1736-43.

66. Miller WC, Koceja DM, Hamilton EJ. A meta-analysis of the past 25 years of weight loss research using diet, exercise or diet plus exercise intervention. Int J Obes Relat Metab Disord. 1997;21 (10):941-7.

67. Pimenta NM, Santa-Clara $H$, Sardinha LB, Fernhall $B$. Body fat responses to a 1-year combined exercise training program in male coronary artery disease patients. Obesity (Silver Spring). 2013;21 (4):723-30. 